\title{
Expression and Clinical Significance of the NCAPH, AGGFI, and FOXC2 Proteins in Serous Ovarian
}

\section{Cancer}

\author{
Yingying Sun $\mathbb{D}^{1, *}$ \\ Xuan Wang ${ }^{1, *}$ \\ Hexin Wen ${ }^{2}$ \\ Bo Zhu ${ }^{1,3}$ \\ Lan $\mathrm{Yu}^{1,3}$
}

'Department of Pathology, The First Affiliated Hospital of Bengbu Medical College, Bengbu, People's Republic of China; ${ }^{2}$ Department of Gastrointestinal Surgery, The First Affiliated Hospital of Bengbu Medical College, Bengbu, People's Republic of China; ${ }^{3}$ Bengbu Medical College, Anhui Key Laboratory of Infection and Immunology, Bengbu, People's Republic of China

*These authors contributed equally to this work
Correspondence: Lan Yu

Department of Pathology, The First Affiliated Hospital of Bengbu Medical University, 287, Changhuai Road, Bengbu, Anhui Province, People's Republic of China

Tel +86- 18555520163

Email yulan790210@163.com
Purpose: Recurrence and metastasis are the most common causes of high mortality rates in patients with serous ovarian cancer (SOC). Non-structural maintenance of chromosomes (non-SMC) condensin I complex subunit H (NCAPH) is a newly identified essential oncoprotein whose function in SOC pathogenesis has not been reported yet. Angiogenic factor with $\mathrm{G}$ patch and FHA domains 1 (AGGF1) is an effective promoter of angiogenesis in humans, leading to cancer cell infiltration and progression. Forkhead box C2 (FOXC2) plays a pivotal role in epithelial-to-mesenchymal transition (EMT). The present study analyzed the correlations among the expressions of these three proteins and their relationships with the clinicopathological characteristics and survival of patients with SOC.

Patients and Methods: The expressions of NCAPH, AGGF1, and FOXC2 were detected by the immunohistochemical examination of 153 SOC tissue samples and 30 serous ovarian cystadenoma tissue samples. Clinicopathologic and follow-up data of the patients were collected. Results: The expressions of NCAPH, AGGF1, and FOXC2 were remarkably higher in the SOC tissue samples than in the serous ovarian cystadenoma tissue samples. The protein expressions were positively correlated with the histological tumor grade, the International Federation of Gynecology and Obstetrics (FIGO) stage, lymph node metastasis, and intraperitoneal implantation, but were negatively correlated with the overall survival (OS). Moreover, multivariate analysis showed that the NCAPH, AGGF1, and FOXC2 expressions, FIGO stage, and histological tumor grade were independent adverse prognostic factors for OS in patients with SOC.

Conclusion: The results of this study show that the expressions of NCAPH, AGGF1, and FOXC2 are promising biomarkers and possible therapeutic targets in patients with SOC.

Keywords: serous ovarian cancer, NCAPH, AGGF1, FOXC2, cancer prognosis

\section{Introduction}

Ovarian cancer is a common malignant neoplasm and the second most lethal gynecological cancer after cervical cancer. It is estimated that more than 310,000 women worldwide were diagnosed with ovarian cancer in 2020, and nearly 210,000 people died of the disease. ${ }^{1}$ Epithelial ovarian cancer (EOC) is the most prevalent subtype of ovarian cancer, accounting for more than $90 \%$ of ovarian carcinomas. EOC can be divided into many histological subtypes, of which serous ovarian cancer (SOC) is the most common and has the worst prognosis. Most patients with SOC are diagnosed in the advanced stages, ${ }^{2}$ often presenting with abdominal metastasis and ascites and have a 5-year overall survival (OS) of approximately 
$48 \%{ }^{3}$ Tumor invasion and metastasis are the major complications in the treatment of ovarian cancer. Therefore, it is of great significance to actively explore novel predictors and innovative therapeutic targets for patients with SOC.

Condensin, a type of multiprotein complex, is involved in chromosomal condensation and segregation during mitosis. ${ }^{4,5}$ There are two types of DNA-condensing protein complexes in most eukaryotic cells: condensin I and condensin II. Non-structural maintenance of chromosomes (non-SMC) condensin I complex subunit H (NCAPH), also known as BRRN1 or CAP-H, is an essential regulatory subunit of condensin I and a member of the kleisin superfamily of proteins. ${ }^{6}$ Several recent studies have revealed that NCAPH is overexpressed in various types of malignancies and, thus, can be considered as a new potential biomarker and therapeutic target in cancers, such as hepatocellular, prostate, and colon cancer. $^{7-9}$ Intriguingly, a recent bioinformatics analysis based on the Gene Expression Omnibus (GEO) database showed that the NCAPH gene may potentially be associated with ovarian carcinogenesis. However, to our knowledge, there are currently no relevant studies elucidating the clinical relevance and function of the NCAPH protein in the pathogenesis and mechanisms involved in SOC. Angiogenic factor with $G$ patch and FHA domains 1 (AGGF1) was originally discovered by Tian et al in patients with Klippel-Trenaunay syndrome (KTS) in 2004. It is overexpressed in vascular endothelial cells and promotes angiogenesis. ${ }^{10}$ Recent studies $^{11-13}$ have shown that AGGF1 is overexpressed in many types of malignancies, including gastric cancer, hepatocellular carcinoma, and colorectal carcinoma, and is significantly associated to the prognosis of patients. Forkhead box $\mathrm{C} 2$ (FOXC2), an important member of the family of forkhead transcription factors, is an embryonic transcriptional regulatory factor involved in embryonic development, angiogenesis, lymphangiogenesis, metabolism, and epithelial-to -mesenchymal transition (EMT). It has been indicated that the overexpression of $\mathrm{FOXC} 2$ in various metastatic tumors, including gynecological malignant tumors, can induce tumor growth and progression. ${ }^{14}$

This study is the first to investigate NCAPH expression at the protein level in SOC tissues. We explored the relationships between the expressions of the NCAPH, AGGF1, and FOXC2 proteins and the clinicopathological features of patients with SOC. Furthermore, we evaluated the relationships between the expressions of these proteins and the invasion, metastasis, and prognosis of SOC. We also investigated the mechanisms involved in SOC.

\section{Materials and Methods Patients and Tissue Samples}

A total of 153 SOC tissue samples and 30 serous ovarian cystadenoma tissue samples were obtained from patients, none of whom had received chemotherapy, radiotherapy, or any other anti-tumor therapy prior to surgical treatment. Related clinical, pathological, and follow-up data of the patients were also collected. All patients were diagnosed at the Department of Pathology of the First Affiliated Hospital of Bengbu Medical College in Anhui Province between January 2012 and January 2015. OS was calculated from the primary operation date of the patients to their death or December 2019 (range: 5-80 months). The patients' ages ranged from 20 to 72 years (average: $53.0 \pm$ 10.7 years). Tumor staging was assessed based on the 2014 International Federation of Gynecology and Obstetrics (FIGO) ovarian cancer staging system and the corresponding tumor-node-metastasis (TNM) classification. Pathological grading was evaluated using the World Health Organization (WHO) standards. This study was approved by the Committee for Medical Ethics of the Bengbu Medical College (NO.2021052). This study was conducted in accordance with the Declaration of Helsinki. Each patient included in the study signed a written informed consent document. The specific patient characteristics are shown in Table 1.

\section{Tissue Preparation for Immunohistochemical Analysis}

Immunohistochemistry for NCAPH, AGGF1, and FOXC2 was performed on the 153 SOC tissue samples and 30 serous ovarian cystadenoma tissue samples using the Elivision $^{\mathrm{TM}}$ Plus detection kit (Lab Vision, Fremont, CA, USA) according to the manufacturer's instructions. After fixing the tissues in 4\% neutral formaldehyde solution, they were embedded in paraffin. The tissue was consecutively cut into $4-\mu \mathrm{m}$ thick sections and placed onto salinized glass slides, deparaffinized and de-waxed with xylene solution and gradient alcohol, respectively, and washed with phosphate buffer solution (PBS, $\mathrm{pH} 7.2$ ) for $10 \mathrm{~min}$. After antigen repair in citrate buffer $(\mathrm{pH} 6.0)$ at $95{ }^{\circ} \mathrm{C}$ for $30 \mathrm{~min}$, endogenous peroxidase activity inhibition was performed by blocking all tissues with methanol containing $0.3 \%$ $\mathrm{H}_{2} \mathrm{O}_{2}$ for $10 \mathrm{~min}$ at room temperature (RT). After several 
Table I Correlation of NCAPH, AGGFI and FOXC2 with Clinicopathologic Factors in I53 Patients with SOC

\begin{tabular}{|c|c|c|c|c|c|c|c|}
\hline \multirow[t]{2}{*}{ Variable } & \multirow{2}{*}{$\frac{\text { Cases }}{n}$} & \multicolumn{2}{|c|}{ NCAPH } & \multicolumn{2}{|c|}{ AGGFI } & \multicolumn{2}{|c|}{ FOXC2 } \\
\hline & & Positive (\%) & $\mathbf{P}$ & Positive (\%) & $\mathbf{P}$ & Positive (\%) & $\mathbf{P}$ \\
\hline \multicolumn{8}{|l|}{ Age (years) } \\
\hline$<53$ & 76 & $49(63.6 \%)$ & 0.740 & $49(63.6 \%)$ & 0.494 & $40(51.9 \%)$ & 0.747 \\
\hline$\geq 53$ & 77 & $46(60.5 \%)$ & & $53(69.7 \%)$ & & $42(55.3 \%)$ & \\
\hline \multicolumn{8}{|l|}{ Diameter } \\
\hline$<7 \mathrm{~cm}$ & 95 & $58(61.1 \%)$ & 0.864 & $63(66.3 \%)$ & 1.000 & $48(50.5 \%)$ & 0.404 \\
\hline$\geq 7 \mathrm{~cm}$ & 58 & $37(63.8 \%)$ & & $39(67.2 \%)$ & & $34(58.6 \%)$ & \\
\hline \multicolumn{8}{|l|}{ Grade } \\
\hline High-grade serous ovarian cancer & 69 & $73(96.9 \%)$ & $<0.001$ & $74(88.1 \%)$ & $<0.001$ & $65(77.4 \%)$ & $<0.001$ \\
\hline Low-grade serous ovarian cancer & 84 & $22(31.9 \%)$ & & $28(40.6 \%)$ & & $17(24.6 \%)$ & \\
\hline \multicolumn{8}{|l|}{ FIGO stage } \\
\hline I-II & 63 & $18(28.6 \%)$ & $<0.001$ & $26(41.3 \%)$ & $<0.001$ & $10(15.9 \%)$ & $<0.001$ \\
\hline III-IV & 90 & $77(85.6 \%)$ & & $76(84.4 \%)$ & & $72(80.0 \%)$ & \\
\hline \multicolumn{8}{|l|}{ LN metastasis } \\
\hline Yes & 47 & $39(83.0 \%)$ & 0.001 & $42(89.4 \%)$ & $<0.001$ & $37(78.7 \%)$ & $<0.001$ \\
\hline No & 106 & $56(52.8 \%)$ & & $60(56.6 \%)$ & & $45(42.5 \%)$ & \\
\hline \multicolumn{8}{|l|}{ Ascite } \\
\hline Yes & 60 & $33(56.0 \%)$ & 0.234 & $36(61.0 \%)$ & 0.291 & $27(45.8 \%)$ & 0.137 \\
\hline No & 93 & $62(66.0 \%)$ & & $66(70.2 \%)$ & & $55(58.5 \%)$ & \\
\hline \multicolumn{8}{|l|}{ Implantation } \\
\hline Yes & 58 & $47(82.5 \%)$ & $<0.001$ & $47(82.5 \%)$ & 0.001 & $42(73.7 \%)$ & $<0.001$ \\
\hline No & 95 & $48(50.0 \%)$ & & $55(57.3 \%)$ & & $40(41.7 \%)$ & \\
\hline
\end{tabular}

washes in PBS, the sections were incubated with the appropriate goat serum for $30 \mathrm{~min}$ at RT and then incubated with NCAPH rabbit anti-human polyclonal antibody (Thermo Fisher Scientific, Waltham, MA, USA; Cat\# PA5-30467, RRID: AB_2547941), FOXC2 rabbit anti-human polyclonal antibody (Thermo Fisher Scientific; Cat\# PA5-24588, RRID: AB_2542088), and AGGF1 mouse anti-human monoclonal antibody (Affinity Biosciences, Cincinnati, OH, USA; Cat\# DF12109, RRID: AB_2844914) for $1 \mathrm{~h}$ at $37^{\circ} \mathrm{C}$. A polymer enhancer (reagent A) and goat anti-mouse antibody (reagent B) were successively added to the slides at RT. Lastly, freshly prepared diaminobenzidine (DAB) solution was added to the slides, which were then counterstained with hematoxylin, and then dehydrated, air-dried, and mounted.

\section{Immunohistochemical Analysis}

All immunohistochemical staining results were evaluated by two independent senior pathologists who were blinded to the patients' clinicopathological features. Brown granules observed under the microscope indicated a positive expression of the proteins. NCAPH and FOXC2 were mainly expressed in the nucleus, while AGGF1 was localized in the cytoplasm of the SOC cells. The comprehensive score was calculated based on the staining intensity and proportion of positive cells. Ten high-power visual fields were selected randomly to calculate the proportion of positive tumor cells. The positive expression rate was graded as follows: $0(\leq 10 \%), 1(11-25 \%), 2(26-50 \%), 3$ $(51-75 \%)$, and $4(>75 \%)$. The staining intensity for NCAPH, AGGF1, and FOXC2 was scored as 0 (negative), 1 (faint), 2 (moderate), and 3 (strong). The final score was determined by multiplying the positive expression rate grade by the staining intensity score. Scores of $0-2$ were categorized as negative, and scores of 3-12 were categorized as positive.

\section{Statistical Analysis}

All data were analyzed using the IBM SPSS Statistics 22.0 software (IBM Corp., Armonk, NY, USA). The relationships between the clinicopathological variables and the expressions of NCAPH, AGGF1, and FOXC2 were 
analyzed using the Chi-squared test. Spearman's coefficient test was used to investigate the associations among the three proteins. The Kaplan-Meier method was used for univariate survival analysis to assess the effects of NCAPH, AGGF1, or FOXC2 on OS. The Cox regression model was used for multivariate analysis to assess the independent prognostic factors in patients with SOC. Statistical significance was set at $\mathrm{P}<0.05$.

\section{Results}

\section{Associations Between the Expressions of $\mathrm{NCAPH}, \mathrm{AGGFI}, \mathrm{FOXC2}$ and the Clinicopathological Parameters}

Immunohistochemistry showed negative or faint expression of NCAPH in serous ovarian cystadenoma tissue samples. The positive expression rates of NCAPH in the SOC tissue samples and serous ovarian cystadenoma tissue samples were $62.1 \%(95 / 153)$ and $0(0 / 30)$, respectively, yielding a significant difference between the two groups of samples $(\mathrm{P}<0.001$, Figure $1 \mathrm{~A}$ and $\mathrm{B})$. The positive expression rate of NCAPH was positively correlated with the histological tumor grade, FIGO stage, intraperitoneal implantation, and lymph node metastasis (LNM), but not with age, tumor size, or presence of ascites (Table 1).

Among the 153 SOC tissue samples, 102 (66.7\%, 102/ 153) exhibited a positive AGGF1 expression, whereas among the 30 benign ovarian tissue samples, only two $(6.6 \%, 2 / 30)$ displayed a low AGGF1 expression, yielding a significant difference between the two groups $(\mathrm{P}<0.001$; Figure $1 \mathrm{C}$ and D). No significant relationship was observed between the positive expression of AGGF1 and age, tumor size, or presence of ascites. However, the expression of AGGF1 was significantly related to the histological tumor grade, FIGO stage, LNM, and intraperitoneal implantation (Table 1).

High FOXC2 expression was found in most of the cancer samples $(53.6 \%, 82 / 153)$, but not in the control group $(0,0 / 30)$. The positive expression rate of FOXC2 in the SOC tissue samples was dramatically higher than that in the serous ovarian cystadenoma tissue samples $(\mathrm{P}<0.001 ;$ Figure $1 \mathrm{E}$ and $\mathrm{F})$. The expression of FOXC2 was significantly associated with the histological tumor grade, FIGO stage, LNM, and intraperitoneal implantation, but not with age, tumor size, or presence of ascites (Table 1).

\section{Impact of the Expressions of NCAPH, AGGFI, and FOXC2 on the Prognosis of Patients with SOC}

According to the survival analysis, the 5-year OS rate of the 153 SOC patients was $38.7 \%$. Follow-up data showed that in the SOC group, NCAPH-positive patients showed a shorter OS (38.51 \pm 17.67 months) than NCAPH-negative patients (67.33 \pm 11.99 months; $\mathrm{P}<0.05$; Figure 2A). Moreover, the OS was significantly reduced in SOC with AGGF1-positive patients (40.87 \pm 19.2 months) compared with that of with AGGF1-negative patients $(66.55 \pm 12.5$ months; $\mathrm{P}<0.05$; Figure 2B). Similarly, the OS of patients with a high FOXC2 expression (36.82 \pm 16.83 months) was significantly shorter than that of patients with a negative FOXC2 expression (64 \pm 15.31 months; $\mathrm{P}<0.05$; Figure $2 \mathrm{C}$ ).
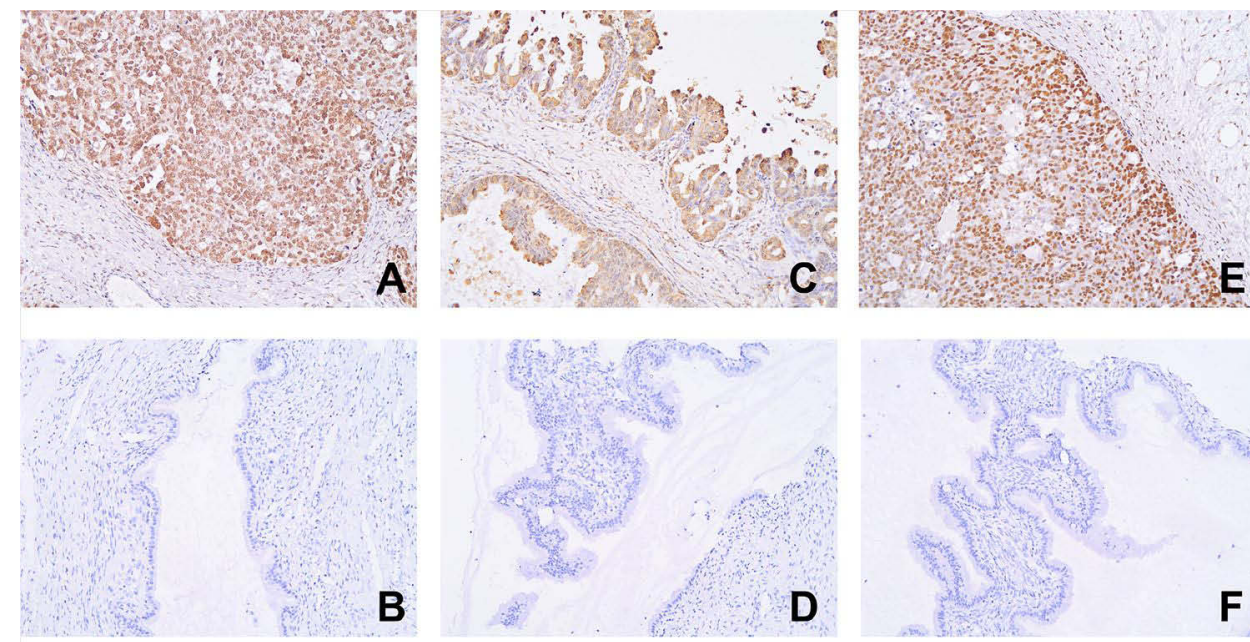

B
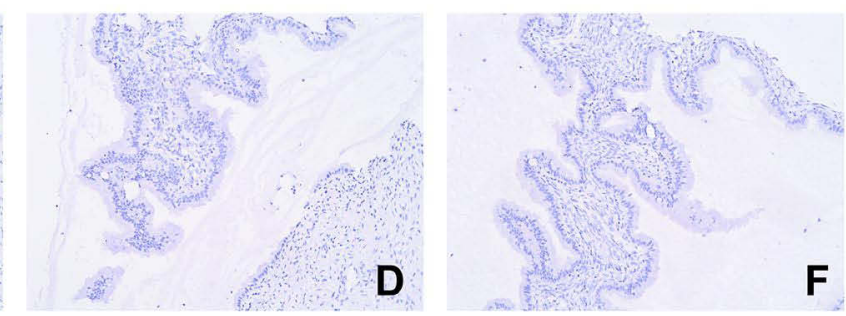

Figure I The expression of the three proteins in SOC and ovarian serous cystadenoma (Elivision ${ }^{\text {TM }}$, $\times 400$; (A) NCAPH in SOC; proportion of positive cells; (B) NCAPH in ovarian serous cystadenoma; (C) AGGFI in SOC; (D) AGGFI in ovarian serous cystadenoma; (E) FOXC2 in SOC; (F) FOXC2 in ovarian serous cystadenoma). 

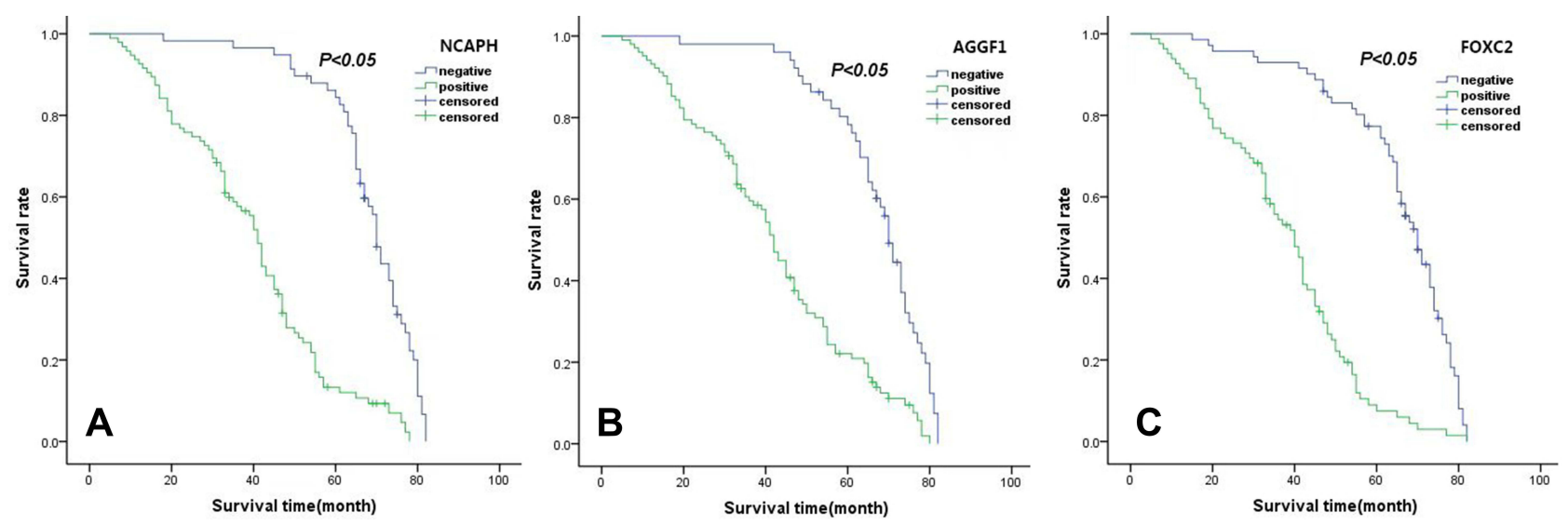

Figure 2 Survival curves of serous ovarian cancer patients ((A) NCAPH, (B) AGGFI, (C) FOXC2).

\section{Correlations Among the Expressions of $\mathrm{NCAPH}, \mathrm{AGGFI}$, and FOXC2 in SOC}

Spearman correlation coefficient analysis showed that NCAPH expression was positively correlated with AGGF1 expression $(\mathrm{r}=0.619, \mathrm{P}<0.001)$ and FOXC2 expression $(\mathrm{r}=0.570, \mathrm{P}<0.05)$. Moreover, AGGF1 expression was positively associated with FOXC2 expression $(\mathrm{r}=0.371, \mathrm{P}<0.001$; Table 2$)$. In SOC patients of stage I-II, NCAPH expression was positively related to AGGF1 $(\mathrm{r}=0.469, \mathrm{P}<0.001)$ and FOXC2 expressions $(\mathrm{r}=0.302, \mathrm{P}<0.001)$, while AGGF1 expression and FOXC2 expression, individually, had no significant correlation $(\mathrm{r}=0.165, \mathrm{P}>0.05$; Table 3). Similarly in SOC patients of stage III-IV, $\mathrm{NCAPH}$ expression was positively related to AGGF1 $(\mathrm{r}=0.521, \mathrm{P}<0.001)$ and FOXC2 expressions $(\mathrm{r}=$ $0.348, \mathrm{P}=0.001$ ), while AGGF1 expression and FOXC2 expression had no significant correlation $(\mathrm{r}=0.092, \mathrm{P}>$ 0.05; Table 4) with each other.

Table 2 Expression of NCAPH, AGGFI and FOXC2 and Their Relationship in SOC

\begin{tabular}{|c|c|c|c|c|c|c|}
\hline \multirow[t]{2}{*}{ Variable } & \multicolumn{2}{|c|}{ NCAPH } & \multirow[t]{2}{*}{$r$} & \multicolumn{2}{|c|}{ AGGFI } & \multirow[t]{2}{*}{$\mathbf{r}$} \\
\hline & Negative & Positive & & Negative & Positive & \\
\hline FOXC2 & & & & & & \\
\hline Negative & 48 & 23 & 0.570 & 37 & 34 & 0.371 \\
\hline Positive & 10 & 72 & & 14 & 68 & \\
\hline AGGFI & & & & & & \\
\hline Negative & 41 & 10 & 0.619 & - & - & - \\
\hline Positive & 17 & 85 & & - & - & \\
\hline
\end{tabular}

Note: All $P<0.001$.

\section{Univariate and Multivariate Analyses}

We then analyzed the factors affecting the prognosis of SOC using the Cox risk regression model. Univariate analysis revealed that NCAPH expression (log-rank = 4.402, $\mathrm{P}<0.05$ ), AGGF1 expression (log-rank $=5.796$, $\mathrm{P}<0.05)$, FOXC2 expression (log-rank $=73.712, \mathrm{P}<$ 0.001 ), the histological tumor grade (log-rank $=92.944$, $\mathrm{P}<0.001)$, FIGO stage (log-rank $=92.234, \mathrm{P}<0.001)$, intraperitoneal implantation (log-rank $=32.766, \mathrm{P}<$ 0.001), and LNM (log-rank $=32.766, \mathrm{P}<0.001$; Table 5) were independent prognostic factors. Multivariate Cox proportional hazard regression analysis revealed that NCAPH, AGGF1, and FOXC2 expressions, FIGO stage, intraperitoneal implantation, LNM, and histological tumor grade were likely independent prognostic factors in patients with SOC $(\mathrm{P}<0.05$; Table 6$)$.

\section{Discussion}

The most common histological subtype of EOC is SOC, characterized by a delayed diagnosis and an extremely low 5 -year survival rate, and gravely threatening the health of women. At present, surgery combined with chemotherapy is the primary treatment for EOC. In recent years, great progress has been made in the research of targeted drugs for ovarian cancer. Two types of targeted drugs, antiangiogenic drugs and poly(adenosine diphosphate-ribose) polymerase inhibitors (PARPIs), are currently available; however, patients with ovarian cancer who are treated with such drugs still face the challenges of recurrence and metastasis. Therefore, the identification of novel targets that can evaluate the biological behavior of SOC and serve as possible therapeutic targets is important. 
Table 3 Expression of NCAPH, AGGFI and FOXC2 and Their Relationship in SOC of Stage I-II

\begin{tabular}{|c|c|c|c|c|c|c|}
\hline \multirow[t]{2}{*}{ Variable } & \multicolumn{2}{|c|}{ NCAPH } & \multirow[t]{2}{*}{$\mathbf{r}$} & \multicolumn{2}{|c|}{ AGGFI } & \multirow[t]{2}{*}{$\mathbf{r}$} \\
\hline & Negative & Positive & & Negative & Positive & \\
\hline \multicolumn{7}{|l|}{ FOXC2 } \\
\hline Negative & 41 & 12 & $0.302^{*}$ & 33 & 20 & $0.165^{* * *}$ \\
\hline Positive & 4 & 6 & & 4 & 6 & \\
\hline \multicolumn{7}{|l|}{ AGGFI } \\
\hline Negative & 33 & 4 & $0.469 * *$ & - & - & - \\
\hline Positive & 12 & 14 & & - & - & \\
\hline
\end{tabular}

Notes: $* \mathrm{P}<0.05 ; * * \mathrm{P}<0.001 ; * * * \mathrm{P}>0.05$.

Table 4 Expression of NCAPH, AGGFI and FOXC2 and Their Relationship in SOC of Stage III-IV

\begin{tabular}{|c|c|c|c|c|c|c|}
\hline \multirow[t]{2}{*}{ Variable } & \multicolumn{2}{|c|}{ NCAPH } & \multirow[t]{2}{*}{$\mathbf{r}$} & \multicolumn{2}{|c|}{ AGGFI } & \multirow[t]{2}{*}{$\mathbf{r}$} \\
\hline & Negative & Positive & & Negative & Positive & \\
\hline \multicolumn{7}{|l|}{ FOXC2 } \\
\hline Negative & 7 & 11 & $0.348^{*}$ & 4 & 14 & $0.092 * * *$ \\
\hline Positive & 6 & 66 & & 10 & 62 & \\
\hline \multicolumn{7}{|l|}{ AGGFI } \\
\hline Negative & 8 & 6 & $0.521^{* *}$ & - & - & - \\
\hline Positive & 5 & 71 & & - & - & \\
\hline
\end{tabular}

Notes: $* \mathrm{P}=0.001 ; * * \mathrm{P}<0.001 ; * * * \mathrm{P}>0.05$

The NCAPH gene, located on chromosome $2 \mathrm{q} 11.2$, is involved in maintaining the stability of lectin complexes and ensuring the accurate isolation of sister chromatids during mitosis; ${ }^{4-6}$ however, little is known about its role in cancer. The function of NCAPH in tumors was first investigated in advanced malignant melanoma in $2007 .{ }^{15}$ It has since been reported that NCAPH is significantly overexpressed in several types of malignant tumor tissues and is involved in the proliferation, invasion, and metastasis of tumor cells. ${ }^{7,9,16,17}$

In the present study, for the first time, we investigated the expression of NCAPH in SOC tissue samples and analyzed its relationship with the clinicopathological characteristics of the patients. We found that the positive expression rate of NCAPH in SOC tissue samples was $62.1 \%$ and was significantly related to the histological tumor grade, FIGO stage, LNM, and intraperitoneal implantation. Patients with NCAPH-positive samples exhibited a later clinical stage, higher histopathological grade, greater tendency for pelvic lymph node metastasis and abdominal metastasis, and significantly shorter OS than those with NCAPH-negative samples, indicating that ovarian cancer with an increased $\mathrm{NCAPH}$ expression demonstrates a stronger invasiveness and results in a worse prognosis than that with a low or absent NCAPH expression. Univariate and multivariate analyses showed that the upregulation of NCAPH can promote SOC invasiveness and metastasis. These results are consistent with the those observed in hepatocellular carcinoma $^{7}$ and oral squamous cell carcinoma, ${ }^{5,11,17}$ but not with those observed in colorectal cancer ${ }^{9}$ and cervical carcinoma, ${ }^{16}$ as patients with colorectal cancer or cervical carcinoma and a positive NCAPH expression exhibited a better prognosis than their counterparts. We speculate that such results were observed because tumors with a positive NCAPH expression have a higher proliferation rate, enhancing the effect of radiotherapy or chemotherapy and improving the prognosis. In this study, patients with SOC who received preoperative radiotherapy, chemotherapy, or other anti-cancer therapies were not selected, and the prognosis was assessed without any treatment factors.

Angiogenesis refers to the formation of new blood vessels from capillaries and arterioles. Tumor angiogenesis is one of the most important factors that promote tumor growth and metastasis. The AGGF1 gene, which is a susceptibility gene for KTS, is located on chromosome 
Table 5 Results of Univariate Analyses of Overall Survival (OS) Time

\begin{tabular}{|c|c|c|c|c|}
\hline Variable & $\mathbf{n}$ & $\begin{array}{l}\text { Mean OS } \\
\text { (Months) }\end{array}$ & $\begin{array}{l}\text { Log- } \\
\text { Rank }\end{array}$ & $\mathrm{p}$ value \\
\hline $\begin{array}{l}\text { NCAPH } \\
\text { Negative } \\
\text { Positive }\end{array}$ & $\begin{array}{l}58 \\
95\end{array}$ & $\begin{array}{l}67.33 \pm 11.99 \\
38.51 \pm 17.67\end{array}$ & 4.402 & 0.036 \\
\hline $\begin{array}{l}\text { AGGFI } \\
\text { Negative } \\
\text { Positive }\end{array}$ & $\begin{array}{l}51 \\
102\end{array}$ & $\begin{array}{l}66.55 \pm 12.5 \\
40.87 \pm 19.2\end{array}$ & 5.796 & 0.016 \\
\hline $\begin{array}{l}\text { FOXC2 } \\
\text { Negative } \\
\text { Positive }\end{array}$ & $\begin{array}{l}71 \\
82\end{array}$ & $\begin{array}{l}64 \pm|5.3| \\
36.82 \pm \mid 6.83\end{array}$ & 73.712 & $<0.001$ \\
\hline $\begin{array}{l}\text { Age } \\
\qquad 553 \\
\geq 53\end{array}$ & $\begin{array}{l}77 \\
76\end{array}$ & $\begin{array}{l}48.65 \pm 21.54 \\
50.22 \pm 20.69\end{array}$ & 0.327 & 0.567 \\
\hline $\begin{array}{l}\text { Diameter } \\
\quad<7 \mathrm{~cm} \\
\geq 7 \mathrm{~cm}\end{array}$ & $\begin{array}{l}95 \\
58\end{array}$ & $\begin{array}{l}49.49 \pm 22.63 \\
49.33 \pm 18.4\end{array}$ & 0.529 & 0.467 \\
\hline $\begin{array}{l}\text { Grade } \\
\text { Hlgh-grade serous } \\
\text { ovarian cancer } \\
\text { Low-grade serous } \\
\text { ovarian cancer }\end{array}$ & 84 & $\begin{array}{l}66.17 \pm 12.89 \\
35.68 \pm 15.88\end{array}$ & 92.944 & $<0.001$ \\
\hline $\begin{array}{l}\text { FIGO stage } \\
\text { I-II } \\
\text { III-IV }\end{array}$ & $\begin{array}{l}63 \\
90\end{array}$ & $\begin{array}{l}68.14 \pm 10.01 \\
36.33 \pm 16.33\end{array}$ & 92.234 & $<0.001$ \\
\hline $\begin{array}{l}\text { LN metastesis } \\
\text { No } \\
\text { Yes }\end{array}$ & $\begin{array}{l}106 \\
47\end{array}$ & $\begin{array}{l}57.15 \pm 18.35 \\
32.02 \pm 15.84\end{array}$ & 44.906 & $<0.001$ \\
\hline $\begin{array}{c}\text { Ascite } \\
\text { No } \\
\text { Yes }\end{array}$ & $\begin{array}{l}94 \\
59\end{array}$ & $\begin{array}{l}48.28 \pm 21.41 \\
51.27 \pm 20.55\end{array}$ & 0.339 & 0.560 \\
\hline $\begin{array}{l}\text { Implantation } \\
\text { No } \\
\text { Yes }\end{array}$ & $\begin{array}{l}96 \\
57\end{array}$ & $\begin{array}{l}57.77 \pm 18.96 \\
35.39 \pm 16.57\end{array}$ & 32.766 & $<0.001$ \\
\hline
\end{tabular}

$5 \mathrm{q} 13$ and encodes an angiogenic factor with a strong angiogenic effect. ${ }^{10}$ AGGF1 maintains vascular integrity and homeostasis by activating the PI3K/AKT signaling pathway, which is indispensable for angiogenesis and the regulation of vascular E-cadherin phosphorylation in vivo and in vitro. ${ }^{18}$ In recent years, increasing evidence ${ }^{11-13}$ has shown that AGGF1 is overexpressed in various malignant tumors, such as hepatocellular carcinoma, colorectal cancer, and gastric cancer, and that AGGF1 expression is positively related to tumor invasion, metastasis, and
Table 6 Multivariate Survival Analysis of Patients with SOC

\begin{tabular}{|l|c|c|c|c|c|}
\hline Covariate & B & SE & RR & $95 \%$ CI & P \\
\hline Age & -0.193 & 0.169 & 0.824 & $0.592-1.148$ & 0.253 \\
Diameter & -0.231 & 0.184 & 0.794 & $0.553-1.139$ & 0.210 \\
Grade & 1.470 & 0.266 & 4.349 & $2.583-7.321$ & $<0.00 I$ \\
FIGO & 0.654 & 0.259 & 1.924 & $1.158-3.198$ & 0.012 \\
NCAPH & 0.635 & 0.277 & 1.887 & $1.097-3.247$ & 0.022 \\
AGGFI & 0.516 & 0.249 & 1.675 & $1.029-2.726$ & 0.038 \\
FOXC2 & 0.581 & 0.236 & 1.787 & $1.125-2.839$ & 0.014 \\
Metastesis & 0.455 & 0.307 & 1.577 & $0.864-2.878$ & 0.138 \\
Ascite & 0.131 & 0.175 & 1.140 & $0.808-1.608$ & 0.454 \\
Implantation & 0.563 & 0.306 & 1.755 & $0.963-3.200$ & 0.066 \\
\hline
\end{tabular}

Abbreviations: B, coefficient of regression; SE, standard error; RR, relative risk; $\mathrm{Cl}$, confidence interval.

TNM stage, rendering AGGF1 a potential independent prognostic factor in patients with such tumors. However, the mechanisms by which AGGF1 promotes tumor angiogenesis vary. In glioblastoma, ${ }^{19}$ angiogenesis can be regulated by the MCM3AP-AS1/miR-211/KLF5/AGGF1 axis, while in hepatocellular carcinoma, ${ }^{20}$ the AKT pathway can be regulated by AGGF1, contributing to angiogenesis and tumor progression. Based on these findings, we proposed that AGGF1 has key effects on the transformation and progression of ovarian cancer cells. We confirmed that the AGGF1 expression in the SOC tissue samples was significantly higher than that in the serous ovarian cystadenoma tissue samples $(\mathrm{P}<0.001)$ and was closely related to the histological tumor grade, FIGO stage, LNM, and intraperitoneal implantation $(\mathrm{P} \leq 0.001)$. Survival analysis revealed that patients with AGGF1-positive samples exhibited a poorer OS than those with AGGF1-negative samples, indicating that ovarian cancer with a high AGGF1 expression demonstrates a stronger invasiveness and results in a worse prognosis than that with a low or absent AGGF1 expression. These results are in line with those of previous reports. ${ }^{11-13}$ Therefore, AGGF1 expression likely plays a crucial role in SOC progression and invasion by promoting angiogenesis.

EMT is a complicated cell reprogramming process that can transform polarized epithelial cells into cells with a mesenchymal-like phenotype, playing essential roles in SOC progression and metastasis. FOXC2, also termed mesenchymal fork frame 1 (MHF1), was first found to be associated with lymphedema-distichiasis syndrome. The FOXC2 gene, located on chromosome 16p24.1, is composed of a single-coding exon and no introns. The 
expression of the FOXC2 transcription factor, which stops after birth, is related to various embryonic tissue differentiation and configuration processes and the formation of blood and lymphatic vessels. Notably, FOXC2 is a necessary transcription factor related to EMT and can induce loss of apical-basal polarity in cells due to reduced E-cadherin, loss of tight junctions and desmosomes, and gain of N-cadherin. As an essential mediator of mesenchymal differentiation in EMT, FOXC2 was identified as a tumor promoter in breast cancer models ${ }^{21}$ and was found to be closely related to tumor proliferation, angiogenesis, and lymphangiogenesis. Moreover, FOXC2 positive expression rates increased significantly with an increase in clinical stage. ${ }^{22}$ Current studies have shown the pivotal importance of FOXC2 in cancer metastasis and prognosis. Studies have also reported the presence of an increased expression of FOXC2 in ovarian cancer. Thus, FOXC2 may be a promising therapeutic target for the treatment of advanced ovarian cancer, particularly cisplatin-resistant ovarian carcinoma. ${ }^{23}$ The PI3K/AKT signaling pathway is a crucial mechanism that helps facilitate the effects of FOXC2 on cancer cell proliferation. As such, the AKT inhibitor LY294002 was shown to inhibit the expression of FOXC2 and cyclin D1, remarkably compromising the growth of cancer cells. ${ }^{14}$ Our study confirmed that FOXC2 was highly expressed in the SOC tissue samples. FOXC2 expression was significantly higher in the SOC group than in the control group $(\mathrm{P}<0.001)$ and was closely related to the histological tumor grade, FIGO stage, LNM, and intraperitoneal implantation $(\mathrm{P} \leq$ 0.001). Survival analysis showed that patients with a high FOXC2 expression exhibited a poorer OS than those with a negative FOXC2 expression, indicating that ovarian cancer with a high expression of FOXC2 demonstrates a stronger invasiveness and results in a worse prognosis than that with a low or absent FOXC2 expression. These results are consistent with previous findings. ${ }^{24-26}$ Therefore, FOXC2 likely plays an important role in SOC transformation and progression and is strongly related to the poor prognosis of patients with SOC.

Although our study showed that NCAPH, AGGF1, and FOXC2 play critical roles in SOC invasion and progression, is it possible that these three proteins interact together? If so, how does it work? The specific mechanism by which the newly identified oncogene NCAPH facilitates oncogenesis remains unclear. This study was the first to jointly detect and analyze the expression of NCAPH, AGGF1, and FOXC2 in ovarian cancer, so as to explore the mechanism by which NCAPH promotes ovarian cancer. This study revealed that, in SOC patients of stage I-II and III-IV, NCAPH expression was positively related to AGGF1 and FOXC2 expressions, while AGGF1 and FOXC2 expression had no significant correlation. As a whole, NCAPH expression was positively correlated with AGGF1 and FOXC2 expressions and AGGF1 expression was positively associated with FOXC2 expression. This discrepancy may be due to the insufficient number of samples. However, these results suggest that there may be a relationship among NCAPH, AGGF1, and FOXC2. NCAPH is closely involved in lymphatic infiltration and LNM through the promotion of lymphatic angiogenesis in oral squamous cell carcinoma. ${ }^{17}$ The positive correlation between NCAPH and AGGF1 indicates that both of them jointly promote the occurrence and development of the disease by promoting vasculogenesis, which further illustrates the importance of NCAPH in angiogenesis.

Similarly, the positive correlation between NCAPH and FOXC2 suggests that both promote ovarian cancer invasion and metastasis through EMT. The activation of EMT is necessary for the distant spread of cancer cells and involves the tight association of NCAPH with the adherens junction pathway. NCAPH is negatively related to the expression of epithelial markers, such as E-cadherin and Slug, but is positively related to the expression of mesenchymal markers, such as $\mathrm{N}$-cadherin and vimentin, particularly in hepatocellular and oral squamous cell carcinoma cells. ${ }^{7,17}$ Recent studies ${ }^{27}$ have found that depletion of NCAPH inhibits ovarian cancer cell activity and invasion and migration ability, and NCAPH gene knockout downregulates EMT-related factors $\mathrm{N}$-cadherin and Vimentin, and upregulates E-cadherin, which is consistent with the results of this study. Therefore, we believe that EMT is an important mechanism by which NCAPH promotes SOC invasion and metastasis. In our study, the positive correlation between NCAPH and FOXC2 expressions confirmed the important role of EMT in SOC. Moreover, NCAPH, collaborating with AGGF1 and FOXC2, contributes to tumor invasion and metastasis by promoting angiogenesis and lymphangiogenesis in SOC.

It is well known that the PI3K/AKT signaling pathway drives tumorigenesis, such as in ovarian cancer, and the activation of this pathway is closely related to the migration, invasion, angiogenesis, and EMT of cancer, making it a highly aggressive cause of cancer. ${ }^{28,29}$ Furthermore, since the positive expression of NCAPH has been shown to be involved in the activation of the $\mathrm{PI} 3 \mathrm{~K} / \mathrm{AKT}$ signaling 
pathway, such as in cervical cancer, ${ }^{16}$ the PI3K/AKT signaling pathway may be important for the three proteins, NCAPH, ACCF1, and FOXC2, in promoting SOC invasion and metastasis. Therefore, NCAPH plays a role similar to AGGF1 and FOXC2 in regulating the PI3K-AKT signaling pathway, and also plays an important role in promoting SOC angiogenesis and EMT. Thus, the specific molecular mechanism by which NCAPH promotes SOC invasion and metastasis must be further elucidated in future research.

Multivariate analysis showed that the expressions of NCAPH, AGGF1, and FOXC2 were independent prognostic factors in patients with SOC, providing a new approach for the development of targeted drugs for patients with SOC. In addition, the prognosis of patients with SOC can potentially be clinically evaluated through the detection of these indicators.

Thus, in this study, we demonstrated that overexpression of NCAPH, AGGF1, and FOXC2 in SOC is associated with angiogenesis and EMT and leads to poor prognosis, suggesting that overexpression of NCAPH, AGGF1, and FOXC2 in SOC may facilitate its susceptibility to invasion and metastasis. Therefore, NCAPH, AGGF1, and FOXC2 can be considered as possible biomarkers for predicting invasion, metastasis and prognosis of SOC.

\section{Conclusion}

This study demonstrated that the NCAPH, AGGF1, and FOXC2 proteins are overexpressed in SOC and that these three proteins may play vital roles in the occurrence, progression, invasiveness, and metastasis of SOC. Furthermore, this study showed that NCAPH, AGGF1, and FOXC2 have the potential to serve as valuable biomarkers in SOC, such that the combined detection of these three proteins may indicate the prognosis of patients with SOC.

\section{Acknowledgments}

This work was supported by the Anhui Nature Science Key Program of College and University (no. KJ2017A224) and Bengbu Medical College innovation plan natural science project (Byycx20065). Yingying Sun and Xuan Wang are co-first authors for this study.

\section{Disclosure}

The authors report no conflicts of interest in this work.

\section{References}

1. Sung H, Ferlay J, Siegel RL, et al. Global cancer statistics 2020: GLOBOCAN estimates of incidence and mortality worldwide for 36 cancers in 185 countries. CA Cancer J Clin. 2021;71(3):209-249. doi: $10.3322 /$ caac. 21660

2. Torre LA, Trabert B, DeSantis CE, et al. Ovarian cancer statistics, 2018. CA Cancer J Clin. 2018;68(4):284-296. doi:10.3322/ caac. 21456

3. De Andrade WP, Da Conceicao Braga L, Goncales NG, et al. HSPA1A, HSPA1L and TRAP1 heat shock genes may be associated with prognosis in ovarian epithelial cancer. Oncol Lett. 2020;19 (1):359-367. doi:10.3892/ol.2019.11095

4. Hirano T. Condensins: universal organizers of chromosomes with diverse functions. Genes Dev. 2012;26(15):1659-1678. doi:10.1101/ gad. 194746.112

5. Wood AJ, Severson AF, Meyer BJ. Condensin and cohesin complexity: the expanding repertoire of functions. Nat Rev Genet. 2010;11 (6):391-404. doi:10.1038/nrg2794

6. Neuwald AF, Hirano T. HEAT repeats associated with condensins, cohesins, and other complexes involved in chromosome-related functions. Genome Res. 2000;10(10):1445-1452. doi:10.1101/ gr. 147400

7. Sun C, Huang S, Wang H, et al. Non-SMC condensin I complex subunit $\mathrm{H}$ enhances proliferation, migration, and invasion of hepatocellular carcinoma. Mol Carcinog. 2019;58(12):2266-2275. doi:10.1002/mc.23114

8. Cui F, Hu J, Xu Z, Tan J, Tang H. Overexpression of NCAPH is upregulated and predicts a poor prognosis in prostate cancer. Oncol Lett. 2019;17(6):5768-5776. doi:10.3892/ol.2019.10260

9. Yin L, Jiang LP, Shen QS, et al. NCAPH plays important roles in human colon cancer. Cell Death Dis. 2017;8(3):e2680. doi:10.1038/ cddis. 2017.88

10. Tian XL, Kadaba R, You SA, et al. Identification of an angiogenic factor that when mutated causes susceptibility to Klippel-Trenaunay syndrome. Nature. 2004;427(6975):640-645. doi:10.1038/nature02320

11. Yao HH, Wang BJ, Wu Y, et al. High expression of angiogenic factor with G-patch and FHA domain1 (AGGF1) predicts poor prognosis in gastric cancer. Med Sci Monit. 2017;23:1286-1294. doi:10.12659/ msm. 903248

12. Tu J, Ying X, Zhang D, et al. High expression of angiogenic factor AGGF1 is an independent prognostic factor for hepatocellular carcinoma. Oncotarget. 2017;8(67):111623. doi:10.18632/oncotarget.22880

13. Zhang X, Sun H, Chen W, He X. Elevated expression of AGGF1 predicts poor prognosis and promotes the metastasis of colorectal cancer. $B M C$ Cancer. 2019;19(1):1252. doi:10.1186/s12885-019-6474-7

14. Wang T, Zheng L, Wang Q, et al. Emerging roles and mechanisms of FOXC2 in cancer. Clin Chim Acta. 2018;479:84-93. doi:10.1016/j. cca.2018.01.019

15. Ryu B, Kim DS, Deluca AM, et al. Comprehensive expression profiling of tumor cell lines identifies molecular signatures of melanoma progression. PLoS One. 2007;2(7):e594. doi:10.1371/journal. pone. 0000594

16. Wang M, Qiao X, Cooper T, et al. HPV E7-mediated NCAPH ectopic expression regulates the carcinogenesis of cervical carcinoma via PI3K/AKT/SGK pathway. Cell Death Dis. 2020;11(12):1049. doi:10.1038/s41419-020-03244-9

17. Shimomura H, Sasahira T, Nakashima C, et al. Non-SMC condensin I complex subunit $\mathrm{H}(\mathrm{NCAPH})$ is associated with lymphangiogenesis and drug resistance in oral squamous cell carcinoma. J Clin Med. 2020;9(1):72. doi: $10.3390 / \mathrm{jcm} 9010072$

18. Zhang T, Yao Y, Wang J, et al. Haploinsufficiency of Klippel-Trenaunay syndrome gene Aggf1 inhibits developmental and pathological angiogenesis by inactivating PI3K and AKT and disrupts vascular integrity by activating VE-cadherin. Hum Mol Genet. 2016;25(23):5094-5110. doi:10.1093/hmg/ddw273 
19. Yang C, Zheng J, Xue Y, et al. The effect of MCM3AP-AS1/miR211/KLF5/AGGF1 axis regulating glioblastoma angiogenesis. Front Mol Neurosci. 2018;10:437. doi:10.3389/fnmol.2017.00437

20. Li W, Fu Q, Man W, et al. LncRNA OR3A4 participates in the angiogenesis of hepatocellular carcinoma through modulating AGGF1/akt/mTOR pathway. Eur J Pharmacol. 2019;849:106-114. doi:10.1016/j.ejphar.2019.01.049

21. Mani SA, Yang J, Brooks M, et al. Mesenchyme Forkhead 1 (FOXC2) plays a key role in metastasis and is associated with aggressive basal-like breast cancers. Proc Natl Acad Sci U S A. 2007;104(24):10069-10074. doi:10.1073/pnas.0703900104

22. Zhou Z, Zhang L, Xie B, et al. FOXC2 promotes chemoresistance in nasopharyngeal carcinomas via induction of epithelial mesenchymal transition. Cancer Lett. 2015;363(2):137-145. doi:10.1016/j. canlet.2015.04.008

23. Li C, Ding H, Tian J, et al. Forkhead box protein $\mathrm{C} 2$ promotes epithelial-mesenchymal transition, migration and invasion in cisplatin-resistant human ovarian cancer cell line (SKOV3/CDDP). Cell Physiol Biochem. 2016;39(3):1098-1110. doi:10.1159/ 000447818

24. Bowers LW, Rossi EL, McDonell SB, et al. Leptin signaling mediates obesity-associated CSC enrichment and EMT in preclinical TNBC models. Mol Cancer Res. 2018;16(5):869-879. doi:10.1158/15417786.MCR-17-0508
25. Borretzen A, Gravdal K, Haukaas SA, et al. FOXC2 expression and epithelial-mesenchymal phenotypes are associated with castration resistance, metastasis and survival in prostate cancer. $J$ Pathol Clin Res. 2019;5(4):272-286. doi:10.1002/cjp2.142

26. Agnihotri NS, Astekar M. The role of novel prognostic markers PROX1 and FOXC2 in carcinogenesis of oral squamous cell carcinoma. J Exp Ther Oncol. 2018;12(3):171-184.

27. Qi Y, Mo K, Zhang T. A transcription factor that promotes proliferation, migration, invasion, and epithelial-mesenchymal transition of ovarian cancer cells and its possible mechanisms. Biomed Eng Online. 2021;20(1):83. doi:10.1186/s12938-021-00919-y

28. Zhou WM, Wu GL, Huang J, et al. Low expression of PDK1 inhibits renal cell carcinoma cell proliferation, migration, invasion and epithelial mesenchymal transition through inhibition of the PI3K-PDK1-Akt pathway. Cell Signal. 2019;56:1-14. doi:10.1016/j. cellsig.2018.11.016

29. Ichikawa R, Kawasaki R, Iwata A, et al. MicroRNA-126-3p suppresses HeLa cell proliferation, migration and invasion, and increases apoptosis via the PI3K/PDK1/AKT pathway. Oncol Rep. 2020;43 (4):1300-1308. doi:10.3892/or.2020.7512

\section{Publish your work in this journal}

Cancer Management and Research is an international, peer-reviewed open access journal focusing on cancer research and the optimal use of preventative and integrated treatment interventions to achieve improved outcomes, enhanced survival and quality of life for the cancer patient.
The manuscript management system is completely online and includes a very quick and fair peer-review system, which is all easy to use. Visit http://www.dovepress.com/testimonials.php to read real quotes from published authors. 